\title{
Comparative Evaluation of Three Different Types of Tooth Brush on Surface Abrasion of Enamel \& Nanohybrid Composite - An in Vitro Study
}

\author{
Dr. Mayurika V. Dabhi ${ }^{1}$, Dr. Kishan K.V ${ }^{2}$, Dr. Siddharth Shah $^{3}$ \\ ${ }^{I}$ Department Of Conservative Dentistry \& Endodontics, K.M Shah Dental College \& Hospital, Vadodara, \\ Gujarat, India \\ ${ }^{2}$ Department Of Conservative Dentistry \& Endodontics, K.M Shah Dental College \& Hospital, Vadodara, \\ Gujarat, India \\ ${ }^{3}$ Department Of Conservative Dentistry \& Endodontics, K.M Shah Dental College \& Hospital, Vadodara \\ Gujarat, India
}

\begin{abstract}
To evaluate \& compare the ability of different tooth brush systems on the surface abrasion of enamel \&Nano-hybrid composite.

Methods and Material: Thirty five freshly extracted, sound, human maxillary premolar teeth were collected for this study \& Class $V$ cavities were prepared on the samples \& restored with Nanohybrid composite. All the samples were mounted, on separate acrylic bases followed by profilometric analysis. The surface roughness was measured using the profilometer. The specimen were divided into 4 groups, wherein, Group 1 $(n=10)$ specimens were brushed with flat ended bristles toothbrush; Group 2(n=10) brushed with zig-zag bristles tooth brush , Group 3 (n=10)with powered tooth brush \& Group4 (n=5) was taken as control group. A commercially available dentifrice was used throughout the study. A single specimen was brushed for 2 times daily for 2 min period for 1 week using a customized brushing apparatus. The pre- and post-roughness value change were analyzed and recorded \& were analused using Wilcoxon, Kruskal Wallis \& Mann Whitney U test.

Results: There was no statistical significant difference seen on enamel \& on composite in all the four groups that is, all the three groups performed equally without any statistical significant difference.
\end{abstract}

Keywords: Abrasion, bristles, dentifrice, toothbrush

\section{Introduction}

Resin composite restorations have begun to constitute a significant portion of dentists' routine practice due to patients' great demand for an aesthetic appearance, and this is possible due to the rapid development of new restorative materials, allowing their use in both anterior and posterior restorations ${ }^{[1]}$.However, certain clinical problems associated with the use of direct composite resins have been seen. Among them there is the surface roughness of the restoration ${ }^{[2]}$. A rough surface increases plaque retention, which may then result in gingival inflammation, superficial staining, secondary caries, and colour change. On the contrary, smooth, highly polished restorations have been shown to be more easily maintained than restorations with rougher surfaces ${ }^{[3,4]}$.

Ever since its invention, the toothbrush has played a vital role in the maintenance of oral health and dental hygiene. The use of toothbrush with dentifrice improves the mechanical control of dental plaque. However, if brushing of the teeth is not done judiciously, it could results in trauma to the soft tissue as well as hard tissue of the oral cavity. ${ }^{[5,6]}$

The performance of a composite material is also dependent upon the fillers it employs. Generally, a composite that has smaller particles is more publishable and retains its polish better than one containing larger particles. Also, generally a composite with a higher filler loading provides stronger mechanical properties. Refinements in the particle size through enhanced milling and grinding techniques resulted in composites with particles that were sub-micron, typically averaging about $0.4-1 \mu \mathrm{m}$. These materials are generally considered to be universal composites (Nano-hybrid) as they can be used for most anterior and posterior applications based on their combination of strength and polish ability ${ }^{[7]}$

The wearing of the tooth surface caused by friction of tooth with a foreign object is called "abrasion." Buccal surfaces of teeth are more prone to abrasion due to overzealous brushing ${ }^{[8]}$. Abrasion is most commonly associated with toothbrushing on the cervical margins of teeth. Various studies have shown that different variables influence toothbrush abrasion. These variables include brushing technique, force of brushing, duration and frequency of brushing, and type of brush, in particular filament stiffness. ${ }^{[9]}$

There are various types of toothbrushes with different bristle design available in the market. Depending on the diameter of the bristles, toothbrushes have been categorized as soft $(0.2 \mathrm{~mm})$, medium $(0.3 \mathrm{~mm})$ and hard 
$(0.4 \mathrm{~mm}) .{ }^{[10,11]}$ In general, toothbrush bristles vary in size and design as well as in length, hardness and arrangement. The stiffness of bristles varies based upon its diameter, length, number of filaments in a tuft, and curvature of filaments. ${ }^{[12]}$

Numerous studies have reported on the abrasion of composite resins ${ }^{[13,14,15]}$. Most of these studies evaluated the induced surface roughness with profilometers, as surface roughness has been used as a criterion to assess and predict the deterioration of restorations of different material types ${ }^{[16]}$. The most commonly used parameter to describe surface roughness is $\mathrm{Ra}$, which is the arithmetic mean of vertical departure of a profile from the mean line ${ }^{[17]}$.

Although the surface finish of composites has been widely investigated both in vitro and in vivo ${ }^{[18]}$, information about the quality of surface finish from different polishing systems \& different toothbrushes on the surface roughness of Nano-hybrid composite resin is scarce and limited.

Therefore, the purpose of this in vitro study is to investigate the influence of different toothbrush systems on the surface roughness of enamel \& Nano-hybrid composite resin.

\section{Materials \& methodology}

Study was conducted in the Department of Conservative dentistry and Endodontics, K.M.Shah Dental College, after ethical clearance. Total 35 sound permanent maxillary $1^{\text {st }}$ premolars were collected from Dept of Oral and Maxillofacial Surgery, KMSDCH. All samples were cleaned by using ultrasonic scaler. All teeth were examined to assure the absence of cracks or defects \& stored in $0.5 \%$ chloramine T. All the teeth were prepared with Class V cavities with a no 329 cylindrical fissure bur under water spray. The gingival margin of the cavity were $1 \mathrm{~mm}$ above the cementoenamel junction (CEJ). The cavity dimensions were $3 \mathrm{~mm}$ in length, $3 \mathrm{~mm}$ in width and $1.5 \mathrm{~mm}$ in depth. The self etch $3 \mathrm{M}$ Scotchbond etchant was applied for 15 seconds using applicator tip. The tooth was rinsed and the excess water was blotted leaving the tooth moist. Using a fully saturated brush tip for each coat, 2 consecutive coats was applied of Single Bond adhesive to enamel and dentin The bonding agent was dried for 2-5 seconds and light cured for 10 secs. Cavity was filled using a single increment of the nanohybrid composite (Filtek Z250 XT, 3M ESPE, USA), a preselected transparent matrix (KERR) was applied over the restoration and cured for 20 secs using the continuous curing cycle $(800 \mathrm{~mW} / \mathrm{cm} 2)$. The labial surface was selected and subjected to toothbrushing cycle.

Toothbrushing model:-

A custom made brushing apparatus was constructed. The brushing apparatus was designed to deliver uniform force and uniform unidirectional motion to the tooth surface. This customized brushing apparatus consists of the following parts:

- Motor: To deliver a uniform force and move toothbrush.

- Handle: To which toothbrush can be attached.

- Base: To support the whole apparatus.

The specimens were then mounted, on separate acrylic bases. The acrylic was allowed to dry completely. Three different commercially available toothbrushes with different bristle orientations (flat trim, zig-zag and bi-level) was used in the study. Toothbrushes were mounted on the brushing apparatus and fixed firmly, so that there was no lateral movement during brushing. The apparatus was so designed that it will facilitate easy replacement of one type of toothbrush with another. The mounted specimens were firmly fixed in position during brushing. The mounted specimens were divided into four groups:

- Group 1:- specimens will be brushed with flat trim bristle design

- Group 2:- specimens will be brushed with zig- zag pattern

- Group 3:- specimens will be brushed with mechanized powered toothbrush

- Group 4:- control group- no toothbrushing cycle was carried out

The brushing regimen was carried out. Single specimen was brushed for 2 min period, twice daily, for 1 week\& one week period is used for the standardization. After the brushing regimen was over, the surface roughness of all the 35 mounted specimens were calculated using profilometer. Profilometric analysis of all the samples was carried out before \& after toothbrushing cyle.

Profilometric analysis:-

Surface roughness test was performed with a contact profilometer (perthometer, mitutoyo, $\mathrm{Sj}-201 \mathrm{p}$ ). It had a diamond tip stylus with tip diameter $2 \mu \mathrm{m}$. Three successive measurements in different directions was recorded for all specimens in each group, and the average surface roughness (Ra) value was obtained.

\section{Observations \& Results}

In group 1, group $2 \&$ group 4, there was no statistical significant difference seen between the surface abrasion on enamel \& on composite before $\&$ after the toothbrushing cycle whereas group 3 showed statistical 
significant difference between the surface abrasion on enamel \& on composite before $\&$ after the toothbrushing cycle.

Table 1: Wilcoxon Signed Rank test

\begin{tabular}{|c|c|c|c|}
\hline \multirow{3}{*}{ P value } & Groups & On enamel & On Composite \\
\cline { 2 - 4 } & Group 1 & .333 & .386 \\
\cline { 2 - 4 } & Group 2 & .386 & .386 \\
\cline { 2 - 4 } & Group 3 & .646 & .114 \\
\hline
\end{tabular}

When inter group comparison was done using Mann Whitney $U$ test it was seen that, there was no statistical significant difference found when all the groups were compared.

Table 2: Intergroup comparison using Mann Whitney U Test

IV. Discussion

\begin{tabular}{|c|c|c|c|}
\hline \multirow{4}{*}{ p- value } & Groups & On enamel & On composite \\
\cline { 2 - 4 } & $1-4$ & .391 & .111 \\
\cline { 2 - 4 } & $1-2$ & .427 & .570 \\
\cline { 2 - 4 } & $1-3$ & .762 & .096 \\
\cline { 2 - 4 } & $2-3$ & .545 & .130 \\
\cline { 2 - 4 } & $2-4$ & .221 & .050 \\
\hline
\end{tabular}

Abrasive wear arises when a hard, rough surface slides against a softer one, digs into it, plows a series of grooves, and causes material loss in the form of loose fragments. The speed with which the material is cut away depends on the shape, hardness, and size of the abrasive particles, as well as on the speed of the movement $\&$ the pressure applied.

Numerous toothbrushes of various designs and specifications are available today for the removal of bacterial plaque and food debris. For the most part, the choice of a toothbrush is a personal decision based on individual preferences. There are various designs of toothbrushes available in Indian market, and over decades they have undergone technical modifications also but still there is no data available equivocally that one design is better than the other. Simultaneously, the injudicious use of these toothbrushes can cause trauma and mechanical wear of the tooth surface. ${ }^{20}$ Various types of toothbrushes available in the market keep the buyer in a state of dilemma as to which one to choose, due to lack of information about the "quality" of it. Various epidemiological surveys conducted in Indian population have shown that a greater proportion of Indian population use medium to hard toothbrush. ${ }^{21,22,23}$

The authors are of the opinion that the lack of knowledge and awareness regarding the correct selection might be the cause for the majority of them to select incorrect toothbrush without knowing the adverse outcomes. Various studies have shown that medium and hard toothbrushes cause higher proportion of abrasions compared with soft toothbrushes. The surface abrasion can be evaluated using a profilometer. ${ }^{24,25}$

Various studies have used profilometric analysis to evaluate change in surface roughness. The Ra of a specimen is the arithmetic average height of roughness component irregularities from the mean line measured within the sampling length. The recordings of profilometer were made by placing the tip of the profilometer at the center of composite restoration of each mounted specimen which was embedded in acrylic base. ${ }^{26,27,28}$

Various studies have shown that different variables influence toothbrush abrasion. These variables include brushing technique, force of brushing, duration and frequency of brushing, and type of brush, in particular filament stiffness. In the present study the brushing technique, brushing force, duration and frequency of brushing were kept constant by construction of a customized brushing apparatus that helped to deliver uniform force. Various studies have recommended the use of customized brushing apparatus to assess the role of toothbrush and toothpaste in abrasion process. ${ }^{29,30}$

Most cyclic models used an alternating treatment with equal numbers of erosive and abrasive challenges, which means that each erosive challenge is followed by brushing, irrespective of the waiting period applied. Only a few in vitro studies used a different setup, in which the samples were subjected to 6 erosive challenges but only 2 brushing cycles/day ${ }^{31,32,33}$ In addition, three in situ studies modified the alternating treatments in such a way that fewer abrasive than erosive challenges were performed each ${ }^{34,35}$. This approach might reflect the clinical situation better, as most people brush their teeth twice daily rather than after each contact with erosive foods or beverages ${ }^{36}$. Hence, in the present study, all the samples were brushed twice daily for 7 days.

In this study, the surface abrasivity produced on the mounted enamel specimen produced by three different toothbrush bristle design was evaluated. The bristle diameters for all the three designs of toothbrushes were same (medium) and in order to minimize differences, toothbrushes of same company (Oral-B) were 
selected. In order to minimize surface abrasivity caused by use of different dentifrices, standardized dentifrice was used in all cases. ${ }^{37}$

Dentifrices have been used in conjunction with toothbrushes since a long time. The use of toothbrush with dentifrice improves the mechanical control of dental plaque. Various studies have found that some degree of abrasivity is needed in toothpaste if satisfactory cleaning of the teeth is to be achieved. On the contrary, some studies have found that toothpaste does not have any contributing effect in the mechanical plaque removal. Besides, regular tooth brushing with dentifrices has been considered an etiological factor in gingival recession and tooth wear as reported by various studies.

The results of current study showed that surface abrasion was produced on each specimen, in all the three groups, which were subjected to brushing cycle. \& it was found that there was no statistical significant difference was seen when all the four groups were compared. The results of the present study were in accordance with the study conducted by Hine, \& it was found that there was no differences between different toothbrush bristle designs ${ }^{37}$. The results were also in accordance to the findings of Sripriya and Shaik Hyder Ali wherein their study, they found that that there is no single superior design of manual toothbrush. Though minor and some site differences in favor of the brushes were seen, they were not statistically significant differences observed $^{38}$.

Irrespective of type of bristle design, surface abrasion was produced in all the three groups. However, the flat trim bristle design was found to be relatively safe compared to other designs. Toothbrush design, brushing frequency, brushing pressure and abrasivity of dentifrice, all affect the degree of cervical abrasion observed among individuals. However, in this study only the bristle design was evaluated by keeping other factors constant. This type of, in vitro study, helps in evaluating surface abrasion caused due to different bristle design before planning expensive and time consuming clinical trials.

Unlike other studies conducted by Dyer et al., FV Teche et al. ${ }^{29}$, in which dental acrylic were used to assess abrasion, the present study had the advantage of using mounted specimens with buccal surface brushed with automated brushing machine. It is believed that there are differences in wear of enamel and dentin and enamel being the first layer of tooth are exposed first to tooth brushing. Thus, enamel needs to be protected. Therefore, the study findings will better demonstrate the role of toothbrush and toothpaste in abrasion process. The study used an especially constructed automated brushing apparatus and every care was taken that the tension was adjusted periodically so that the machine delivered uniform force.

However, this in vitro study had certain limitations. One of the factors that could be of much importance in methodological resemblance of the dental abrasion in vitro researches to its really occurring situation inside the mouth is the simulation of continuous washing action of the saliva and its remineralizing protective effects over the worn surfaces of teeth. Few in vitro studies have been conducted assessing the role of saliva in abrasion and it was concluded that the abrasion was significantly lowered if saliva was used as a medium. In the present study, the effect of saliva and its role in prevention of abrasion was not taken into consideration. Saliva is essential for a lifelong conservation of the dentition. ${ }^{39}$ Previous studies carried out by Kumar et al., Hila Hajizadeh et al., Zuryati et al., Kaur and Nandlal have evaluated abrasion produced on dental materials but these studies also had the limitation that the plausible role of saliva in abrasion process was not evaluated. ${ }^{40,41,42}$

Abrasion is of multifactorial etiology and numerous factors affect the abrasion process \& there are still probabilities that numerous other factors may directly or indirectly influence abrasion process. This in vitro study was performed for a short duration; hence, the role of toothbrush and toothpaste for long term use cannot be documented. Moreover, the study did not take into consideration the abrasive nature of toothpastes. Thus, further studies with varying abrasive nature of dentifrices is recommended to assess variation in abrasion process with varying abrasivity of dentifrices. Considering the limitations of in vitro studies, further research supported by in vivo studies need to be conducted before the results can be generalized.

\section{Conclusion}

Thus, from the results of the present study it can be concluded that all the tooth brush designs performed equally in causing tooth surface abrasion. However, further long-term longitudinal and clinical studies are needed to clarify the relationship of the different designs of toothbrush bristles and soft- and hardtissue abrasion.

\section{References}

[1]. Cesar P and Regina $\mathrm{G}$ et al .Surface roughness and color change of a composite: Influence of beverages and brushing . Dental Materials Journal 2012; 31(4): 689-696.

[2]. Gabrielle R, Nathalia c, Soraia d, Antonio , Erick c, Ivone. Effect of Different Finishing/Polishing Systems on the Surface Roughness of Two Heat-Treated Composite Resins Materials Research. 2011; 14(2): 142-145.

[3]. Strassler HE, Bauman G. Current concepts in polishing composite resins. PractPeriodonticsAesthet Dent 1993; 5: 12-17.

[4]. Weitman RT, Eames WB. Plaque accumulation on composite surfaces after various finisingprocedures.J Am Dent Assoc 1975; 91 : 101-106. 
[5]. Ebenezer J, Adhikari DD, Mathew GC, Chacko RK. An unusual injury from a toothbrush: a case report.J Indian Soc Pedod Prev Dent. 2007;25:200-202.

[6]. Parizotto SP, Rodrigues CR, Singer Jda M, Sef HC. Effectiveness of low cost toothbrushes, with or without dentifrice, in the removal of bacterial plaque in deciduous teeth. Pesqui Odontol Bras. 2003;17:17-23

[7]. Jack L. FerracaneReviewResin composite- State of the art. Dent Mater. 2011 Jan;27(1):29-38.

[8]. Grippo JO, Simring M, Schreiner S. Attrition, abrasion, corrosion and abfraction revisited: a new perspective on tooth surface lesions. J Am Dent Assoc. 2004;135:1109-18.

[9]. Bartlett DW, Shah P. A critical review of non-carious cervical (wear) lesions and the role of abfraction, erosion, and abrasion. J Dent Res. 2006;85:306-12.

[10]. Carranza FA. Clinical Periodontology. 9th ed. St-Louis, Missouri: Saunders; 2003. pp. 651-4.

[11]. Wilkins EM, McCullough PA. Clinical Practice of the Dental Hygienist. 3rd ed. Philadelphia: Lea and Febiger; 1999. pp. $277-355$.

[12]. Baseren M. Surface roughness of nanofill and nanohybrid composite resin and ormocer-based tooth-colored restorative materials after several finishing and polishing procedures. J BiomaterAppl 2004; 19: 121-134.

[13]. Turkun LS, Turkun M. The effect of one-step polishing system on the surface roughness of three esthetic resin composite materials. Oper Dent 2004 29: 203-211.

[14]. Lutz F, Setcos JC, Phillips RW. New finishing instruments for composite resins. J Am Dent Assoc1983; 107: 575-580.

[15]. Smales RJ, Webster DA, Leppard PI. Predictions of restoration deterioration. J Dent 1992; 20: 215-220.

[16]. Setcos JC, Tarim B, Suzuki S. Surface finish produced on resin composites by new polishing systems. Quintessence Int 1999; 30: $169-173$.

[17]. Hoelscher D, Neme A, Pink FE, Hughes PJ. The effect of three finishing systems on four esthetic restorative materials. Oper Dent 1998; 23: 36-42.

[18]. Hondrum S, Fernández R. Contouring, finishing and polishing Class 5 restorative materials. Oper Dent 1997; 22: 30-36.

[19]. Alexander JF, Saffir AJ, Gold W. The measurement of the effect of toothbrushes on soft tissue abrasion. J Dent Res 1977;56:722-7.

[20]. Sasan D, Thomas B, Mahalinga BK, Aithal KS, Ramesh PR. Toothbrush selection: a dilemma? Indian J Dent Res 2006;17:167-70.

[21]. Madhan Kumar S, Singarampillay V, Natrajan S. Oral hygiene awareness among two non professional college students in Chennai, India - A pilot study. Int J Sci Res Publ 2012;2:1-5.

[22]. Jain N, Mitra D, Ashok KP, Dundappa J, Soni S, Ahmed S. Oral hygieneawareness and practice among patients attending OPD at Vyas Dental College and Hospital, Jodhpur. J Indian Soc Periodontol 2012;16:524-8.

[23]. Carvalho Rde S, Rossi V, Weidlich P, Oppermann RV. Comparative analysis between hard-and soft-filament toothbrushes related to plaque removal and gingival abrasion. J Clin Dent 2007;18:61-4.

[24]. Zanatta FB, Bergoli AD, Werle SB, Antoniazzi RP. Biofilm removal and gingival abrasion with medium and soft toothbrushes. Oral Health Prev Dent 2011;9:177-83.

[25]. Worschech CC, Rodrigues JA, Martins LR, Ambrosano GM. In vitro 8. Worschech CC, Rodrigues JA, Martins LR, Ambrosano GM. In vitro evaluation of human dental enamel surface roughness bleached with $35 \%$ carbamide peroxide and submitted to abrasive dentifrice brushing. Braz Oral Res 2003;17:342-8.

[26]. Willems G, Lambrechts P, Braem M, Vuylsteke-Wauters M, Vanherle G. The surface roughness of enamel-to-enamel contact areas compared with the intrinsic roughness of dental resin composites. J Dent Res 1991;70:1299-305.

[27]. Young AA, Saliba NA, Consani S, Sinhoreti MA. In vitro evaluation of the abrasiveness of a commercial low-abrasive dentifrice and an experimental dentifrice containing vegetable oil. Braz J Oral Sci 2008;7:1526-30.

[28]. Wiegand A, Kuhn M, Sener B, Roos M, Attin T. Abrasion of eroded dentin caused by toothpaste slurries of different abrasivity and toothbrushes of different filament diameter. J Dent 2009;37:480-4.

[29]. Teche FV, Paranhos HF, Motta MF, Zaniquelli O, Tirapelli C. Differences in abrasion capacity of four soft toothbrushes. Int J Dent Hyg 2011;9:274-

[30]. Ganss C, Hardt M, Blazek D, Klimek J, Schlueter N: Effects of toothbrushing force on the mineral content and demineralized organic matrix of eroded dentine. Eur J Oral Sci 2009a;117:255-260.

[31]. Ganss C, Schlueter N, Hardt M, von Hinckeldey J, Klimek J: Effects of toothbrushing on eroded dentine. Eur J Oral Sci 2007b;115:390-396.

[32]. Lagerweij MD, Buchalla W, Kohnke S, Becker K, Lennon AM, Attin T: Prevention of erosion and abrasion by a high fluoride concentration gel applied at high frequencies. Caries Res 2006;40:148-153.

[33]. Magalhaes AC, Wiegand A, Rios D, Hannas A, Attin T, Buzalaf MA: Chlorhexidine and green tea extract reduce dentin erosion and abrasion in situ. J Dent 2009;37:994-998.

[34]. Vieira A, Jager DH, Ruben JL, Huysmans MC: Inhibition of erosive wear by fluoride varnish. Caries Res 2007;41:61-67.

[35]. Ganss C, Schlueter N, Preiss S, Klimek J: Tooth brushing habits in uninstructed adults -frequency, technique, duration and force. Clin Oral Investig 2009b;13:203- 208.

[36]. Bass CC. The optimum characteristics of toothbrushes for personal oral hygiene. Dent Items Interest 1948;70:697-718.

[37]. Hine M. The toothbrush. Int Dent J 1956;6:15-25.

[38]. Sripriya N, Shaik Hyder Ali KH. A comparative study of the efficacy of four different bristle designs of tooth brushes in plaque removal. J Indian Soc Pedod Prev Dent 2007;25:76-81.

[39]. Kon M, Karuta K, Ogura H. Effects of occlusal and brushing forces on wear of composite resins. Dent Mater J. 2006;25:183-94.

[40]. Hu X, Shortall AC, Marquis PM. Wear of three dental composites under different testing conditions. J Oral Rehabil. 2002;29:75664.

[41]. Lu H, Lee YK, Oguri M, Powers JM. Properties of a dental resin composite with a spherical inorganic filler. Oper Dent. 2006;31:734-40.

[42]. Mitra SB, Wu D, Holmes BN. An application of nanotechnology in advanced dental materials. J Am Dent Assoc. 2003;134:138290.

[43]. Heintze SD, Forjanic M. Surface roughness of different dental materials before and after simulated toothbrushing in vitro. Oper Dent. 2005;30:617-26.

[44]. Xing XS, Li RKY. Wear behavior of epoxy matrix composites filled with uniform sized sub-micron spherical silica particles. Wear. 2004;256:21-6.

[45]. Gedik R, Hürmüzlü F, Coskun A, Bektas OO, Özdemir K. Surface roughness of new microhybrid resin-based composites. J Am Dent Assoc. 2005;136:1106-12.

[46]. . Kim KH, Ong JL, Okumo O. The effect of filler loading and morphology on the mechanical properties of contemporary composites. J Prosthet Dent. 2002;87:642-9. 
[47]. Van Nieuw Amerongen A, Bolscher JG, Veerman EC. Salivary proteins: Protective and diagnostic value in cariology? Caries Res 2004;38: 247-53.

[48]. Hajizadeh H, Ameri H, Eslami S, Mirzaeepoor B. The effect of bleaching of toothbrush abrasion of resin composites. J Conserv Dent 2013;16:17-20.

[49]. Zuryati AG, Qian OQ, Dasmawati M. Effects of home bleaching on surface hardness and surface roughness of an experimental nanocomposite. J Conserv Dent 2013;16:356-61.

[50]. Kaur H, Nandlal B. Effect of dietary solvents on the strength of nanocomposite, compomer, glass ionomer cement: An in-vitro study. J Conserv Dent 2013;16:527-31. 